\title{
The Effect of Intravenous Metoclopramide on Pain, Nausea, Discomfort, and Ease of Insertion of Nasogastric Tube in Emergency Department: A Double-blind Randomized Clinical Trial
}

\author{
Seyed Mohammad Hosseininejad', Farzad Bozorgi ${ }^{2 *}$, Asieh Khodami ${ }^{3}$, \\ Mohammad Hajizade Juybari ${ }^{4}$, and Hamed Aminiahidashti ${ }^{5}$ \\ ${ }^{1}$ Department of Emergency Medicine, Diabetes Research Center, Faculty of Medicine, \\ Mazandaran University of Medical Sciences, Sari, Iran \\ ${ }^{2}$ Department of Emergency Medicine, Orthopedic Research Center, Faculty of Medicine, \\ Mazandaran University of Medical Sciences, Sari, Iran \\ ${ }^{3}$ Student Research Committee, Mazandaran University of Medical Sciences, Sari, Iran \\ ${ }^{4}$ Faculty of Medicine, Mazandaran University of Medical Sciences, Sari, Iran \\ ${ }^{5}$ Department of Emergency Medicine, Faculty of Medicine, Mazandaran University of Medical \\ Sciences, Sari, Iran \\ ORCID:
}

Farzad Bozorgi: https://orcid.org/0000-0001-7582-9735

Corresponding Author:

Farzad Bozorgi; email:

drfarzadbozorgi@yahoo.com

Received 02 September 2021

Accepted 05 December 2021

Published 31 December 2021

Production and Hosting by

Knowledge E

(c) Seyed Mohammad Hosseininejad et al.. This article is distributed under the terms of the Creative

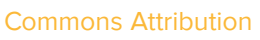

which permits

unrestricted use and

redistribution provided that

the original author and

source are credited.

Editor-in-Chief:

Prof. Mohammad A. M. Ibnouf

\section{Abstract}

Background: Placement of nasogastric tube (NGT) is a routine procedure in the emergency departments, which can be uncomfortable, painful, and cause nausea. The aim of this study was to investigate the effect of intravenous metoclopramide on the ease of NGT insertion, as well as reduction of patients' pain, nausea, and discomfort during NGT insertion in the emergency department.

Methods: In this randomized, double-blind, placebo-controlled trial, 80 patients referred to Imam Khomeini Hospital, Mazandaran Province, Iran were enrolled. Data were collected from December 2015 to March 2016. Participants were selected via convenience sampling and randomly divided into two equal groups (placebo and intervention groups). In metoclopramide and placebo groups, $10 \mathrm{mg}$ of metoclopramide and $10 \mathrm{mg}$ of normal saline solution were administered, respectively. All of the NGT was inserted 15-20 mins after the intravenous infusion. Patient-reported pain, discomfort, and nausea were evaluated using visual analogue scale (VAS), at four time points including before $\left(T_{0}\right)$, immediately $\left(T_{1}\right), 30$ min after $\left(T_{2}\right)$, and 1 hr after the NGT placement $\left(T_{3}\right)$. The ease of NGT insertion was evaluated as easy, moderate, and difficult to pass.

Results: None of the patients had pain, nausea, and discomfort in $\mathrm{T}_{0}$. Additionally, for those who received intravenous metoclopramide, pain intensity significantly decreased compared with the placebo group in $\mathrm{T}_{1}$ (37.7 vs 55.0), $\mathrm{T}_{2}$ (26.2 vs 41.7), and $\mathrm{T}_{3}(20.5$ vs 33.7), respectively $(P<$ 0.001). Nausea intensity decreased significantly over time among patients in the intervention group compared with the placebo group in $\mathrm{T}_{1}$ (32.7 vs 43.2), $\mathrm{T}_{2}$ (19.5 vs 31.2), and $\mathrm{T}_{3}$ (9.0 vs 21.7), respectively $(P<0.001)$. The intensity of patients' discomfort decreased significantly among patients in the intervention group compared with the placebo group in $T_{1}$ (39.5 vs 54.0), $\mathrm{T}_{2}$ (28.7 vs 40.2), and $\mathrm{T}_{3}$ (26.2 vs 39.6), respectively $(P<0.001)$. Patients in the intervention group had easier placement of NGT compared with the placebo group (Easy: $40.0 \%$ vs $0.0 \%$, Moderate: $45.0 \%$ vs $62.5 \%$, and Difficult: $15.0 \%$ vs $37.5 \% ; P<0.001$ ).

Conclusion: Based on the results of the present study, it seems that intravenous metoclopramide can be used as a promising modality for improving the ease of NGT placement and reducing patients' pain, nausea, and discomfort during NGT insertion in the emergency department. 


\section{Introduction}

Placement of nasogastric tube (NGT) is a routine procedure in the emergency departments, which can be uncomfortable, painful, and cause nausea in patients [1]. Although insertion of NGT is usually a simple procedure, it can be complicated without active patient involvement [2]. Improper NGT placement has been associated with some complications such as epistaxis; inadvertent intracranial or tracheobronchial placement of NGT; pharyngeal, esophageal, or bronchial perforation; pneumothorax; and aspiration pneumonia [3,4]. Therefore, appropriate NGT placement strategies can reduce patients' discomfort, pain, and nausea during and after the procedure [5].

Previous research evidence indicated the potential efficacy of metoclopramide in prevention of nosocomial pneumonia and postoperative ileus in patients fed via NGT $[6,7]$. Metoclopramide is a central trigger-zone inhibitor and highly effective in reducing nausea [8]. This medicine has no cardiac side effects and should be intravenously administered 15 to $30 \mathrm{~min}$ before the procedure [9]. A study in Turkey showed that the intravenous administration of metoclopramide reduces patients' pain, discomfort, and nausea during NGT placement [10].

Therefore, due to the importance of this issue, the present study was conducted focusing on the effect of metoclopramide on the ease of NGT insertion, as well as reduction of patients' pain, nausea, and discomfort during NGT insertion in emergency department.

\section{Material and methods}

\subsection{Study design and sample}

In this randomized, double-blind, placebo-controlled trial, 80 patients referred to Imam Khomeini Hospital, Mazandaran Province, Iran were enrolled. Data were collected from December 2015 to March 2016. Participants were selected via convenience sampling and randomly allocated into two equal groups (placebo and intervention).

\subsection{Inclusion and exclusion criteria}

Adult patients older than 18 years, requiring NGT placement in emergency department were included. The exclusion criteria were patients with deformity of the nose and throat, 
allergy to metoclopramide, a history of failure to NGT placement, and hemodynamic instability (systolic blood pressure of $<90 \mathrm{mmHg}$ ).

\subsection{Randomization and blinding}

Patients were randomly allocated to the metoclopramide or placebo groups in a 1:1 ratio. A computer-generated randomization schedule was prepared by the principal investigator who was not otherwise involved in the study. A unique code was assigned to each patient. This code indicated the assigned treatment to each patient and was matched with the study drug syringe. Patients and outcome assessor were blinded to the treatment allocation and were not informed about the randomization procedure. Metoclopramide or normal saline was prepared in the same size syringe and with same volume and appearance.

\subsection{Intervention}

In both groups, intravenous administration of metoclopramide or normal saline were conducted $15 \mathrm{~min}$ before the NGT placement using 2-ml syringes. The syringes were coded by five-digit codes. Patients in metoclopramide and placebo groups received $10 \mathrm{mg}$ (2 ml) of intravenous metoclopramide (Alhavi Pharma Co., Tehran, Iran) or $2 \mathrm{ml}$ of normal saline over 2 min, respectively. Drug or placebo was given or performed by experienced emergency nurses who were interested to cooperate in the study and were blind to the group allocation. They were trained and asked to carefully match the highlighted codes on the study syringes and patients' documents. All of the NGT were inserted 15-20 mins after the intravenous infusion. The NGT placement was performed by two experienced emergency nurses. An appropriate size of NGT was assigned for each of the patients. Also, lubricant gel was used for all patients in both groups to improve the procedure.

\subsection{Outcomes}

Primary outcomes were intensity of patient-reported pain, discomfort, and nausea based on the visual analogue scale (VAS), at four time points including before $\left(\mathrm{T}_{0}\right)$, immediately $\left(T_{1}\right), 30$ min after $\left(T_{2}\right)$, and $1 \mathrm{hr}$ after the NGT placement $\left(T_{3}\right)$. The intensity of pain, discomfort, and nausea of patients, based on the VAS score, were collected thrice at four time points. Based on the VAS, 0 and 100 dictated the lowest and highest felt 
TABLE 1: Clinical and demographic characteristics of participants $(n=80)$

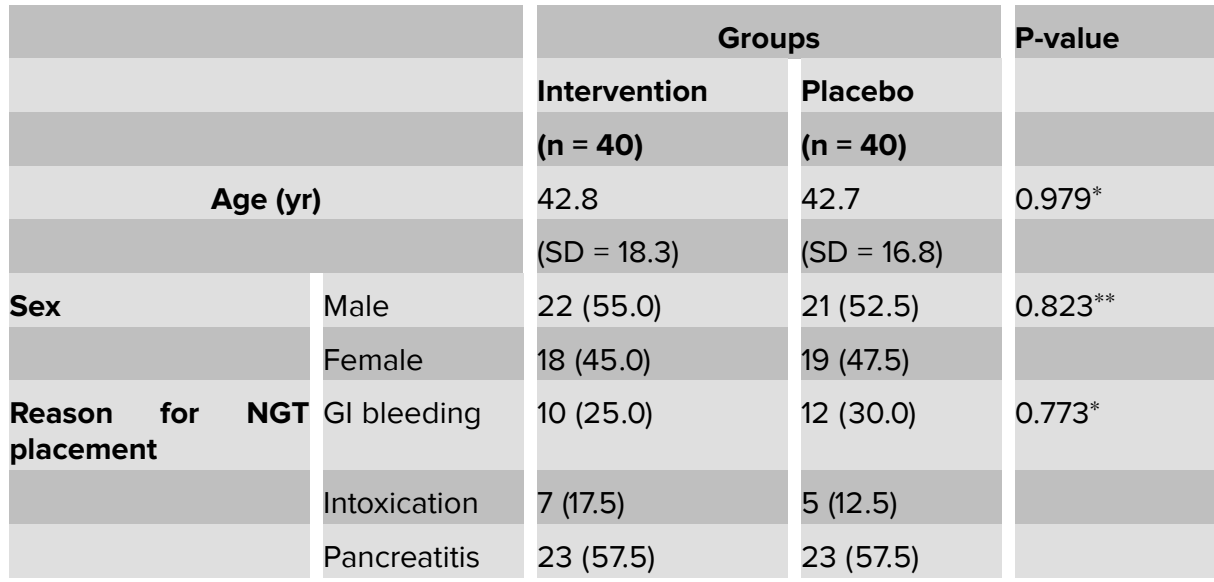

SD: Standard Deviation. Data are presented as number (\%) and mean (SD). ${ }^{*} P$-value obtained using Chi-square test; ${ }^{* *} P$-value obtained with $t$-test.

disturbance by patients. The secondary outcome was the level of difficulty of NGT insertion, which was reported by an emergency nurse as easy, moderate, and difficult to pass.

\subsection{Statistical analysis}

Data were analyzed using the statistical package for social sciences (SPSS v.16.0, SPSS Inc., Chicago, IL, USA). The mean (standard deviation) and number (percentage) were used to show continuous variables. An independent sample $t$-test was applied to compare the means of pain, nausea, and discomfort between metoclopramide and placebo groups. The difficulty of NGT insertion was also compared using the Chi-square test. A $p<0.05$ was considered to be significant.

\section{Results}

\subsection{Participants}

Eighty out of the one hundred and twenty patients who needed an NGT placement were enrolled in the present study (Figure 1).

A total of $55 \%$ and $52.5 \%$ of patients in the intervention and control groups were male with a mean age of $42.8(S D=18.3)$ and $42.7(S D=16.8)$ years, respectively (Table 1). 


\subsection{Outcomes}

Table 2 presents the intensity of pain, nausea, and discomfort of patients, based on VAS score, among placebo and intravenous metoclopramide groups. None of the patients had pain, nausea, and discomfort in $\mathrm{T}_{0}$.

\subsubsection{Pain intensity}

Pain decreased among patients in the intervention group compared with placebo in $\mathrm{T}_{1}$ (37.7 vs 55.0), $\mathrm{T}_{2}$ (26.2 vs 41.7), and $\mathrm{T}_{3}$ (20.5 vs 33.7), respectively $(P<0.001$ ).

\subsubsection{Nausea intensity}

Intensity of patients' nausea decreased significantly among patients in the intervention group compared with placebo in $T_{1}$ (32.7 vs 43.2), $T_{2}$ (19.5 vs 31.2), and $T_{3}$ (9.0 vs 21.7), respectively $(P<0.001)$

\subsubsection{Discomfort}

The intensity of patients' discomfort significantly decreased among patients in the intervention group compared with the placebo group in $T_{1}$ (39.5 vs 54.0), $T_{2}$ (28.7 vs 40.2$)$, and $T_{3}$ (26.2 vs 39.6), respectively $(P<0.001)$

\subsubsection{Level of difficulty of NGT placement}

As presented in Figure 2, patients in the intervention group had easier placement of NGT compared with the placebo group (Easy: $40.0 \%$ vs $0.0 \%$, Moderate: $45.0 \%$ vs $62.5 \%$, and Difficult: $15.0 \%$ vs $37.5 \%$; $P<0.001$ ).

\section{Discussion}

This study assessed the effect of intravenous metoclopramide on the ease of NGT insertion, reduction of pain, nausea, and discomfort in an emergency department. Based on the findings of this study, intravenous metoclopramide had a significant effect on the ease of NGT placement, reduction of pain, nausea, and discomfort in patients who required NGT insertion. 
TABLE 2: Pain, nausea, and discomfort of the patients based on VAS among placebo and metoclopramide groups $(n=80)$

\begin{tabular}{|c|c|c|c|c|}
\hline & & \multicolumn{2}{|c|}{ Groups } & \multirow[t]{2}{*}{ P-value } \\
\hline & & $\begin{array}{l}\text { Intervention ( } \mathrm{n}= \\
\text { 40) }\end{array}$ & Control $(n=40)$ & \\
\hline \multirow[t]{4}{*}{ Pain } & $\mathrm{T}_{0}$ & $0.0(\mathrm{SD}=0.0)$ & $0.0(\mathrm{SD}=0.0)$ & $<0.001$ \\
\hline & $\mathrm{T}_{1}$ & $37.7(\mathrm{SD}=9.9)$ & $55.0(\mathrm{SD}=7.0)$ & \\
\hline & $\mathrm{T}_{2}$ & $26.2(\mathrm{SD}=9.2)$ & $41.7(\mathrm{SD}=6.3)$ & \\
\hline & $\mathrm{T}_{3}$ & $20.5(\mathrm{SD}=9.3)$ & $33.7(\mathrm{SD}=6.2)$ & \\
\hline \multirow[t]{4}{*}{ Nausea } & $\mathrm{T}_{0}$ & $0.0(\mathrm{SD}=0.0)$ & $0.0(\mathrm{SD}=0.0)$ & $<0.001$ \\
\hline & $\mathrm{T}_{1}$ & $32.7(\mathrm{SD}=9.6)$ & $43.2(\mathrm{SD}=7.2)$ & \\
\hline & $\mathrm{T}_{2}$ & $19.5(\mathrm{SD}=9.5)$ & $31.2(\mathrm{SD}=6.8)$ & \\
\hline & $\mathrm{T}_{3}$ & $9.0(\mathrm{SD}=8.5)$ & $21.7(\mathrm{SD}=6.7)$ & \\
\hline \multirow[t]{4}{*}{ Discomfort } & $\mathrm{T}_{0}$ & $0.0(\mathrm{SD}=0.0)$ & $0.0(S D=0.0)$ & $<0.001$ \\
\hline & $\mathrm{T}_{1}$ & $39.5(\mathrm{SD}=10.6)$ & $54.0(\mathrm{SD}=7.0)$ & \\
\hline & $\mathrm{T}_{2}$ & $28.7(\mathrm{SD}=10.4)$ & $40.2(\mathrm{SD}=6.9)$ & \\
\hline & $\mathrm{T}_{3}$ & $26.2(\mathrm{SD}=10.2)$ & $39.6(\mathrm{SD}=6.7)$ & \\
\hline
\end{tabular}

SD: Standard Deviation; T0: Before NGT placement; T1: Immediately after NGT placement; T2: 30 min after NGT placement; T3: 1 hr after NGT placement. Data are presented as mean (SD). $P$-value was obtained with $t$-test.

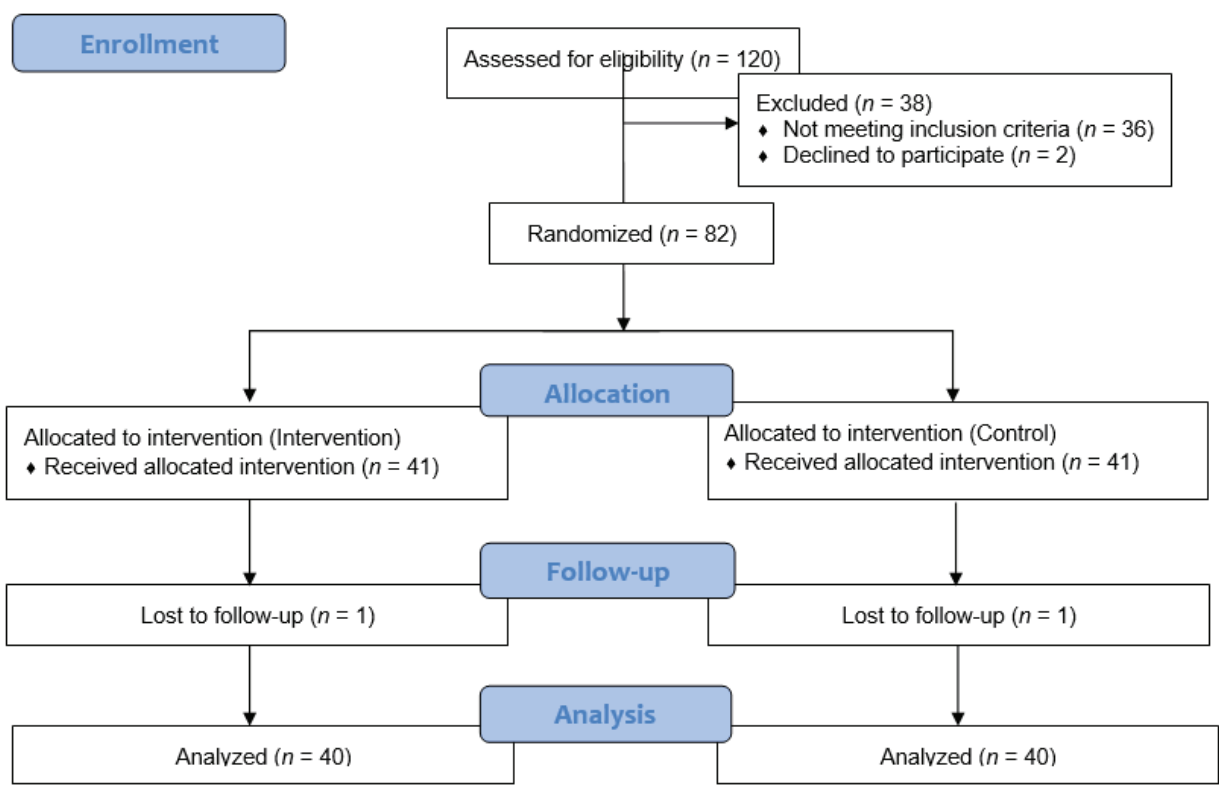

Figure 1: Flowchart of the study.

Previous evidence has shown that NGT placement can be an unpleasant experience among admitted patients in the emergency department [11, 12]. The findings of the present study showed that pain, nausea, and discomfort among patients of the placebo group in $T_{1}, T_{2}$, and $T_{3}$ were 55,43 , and 54 , respectively. These results are relatively 


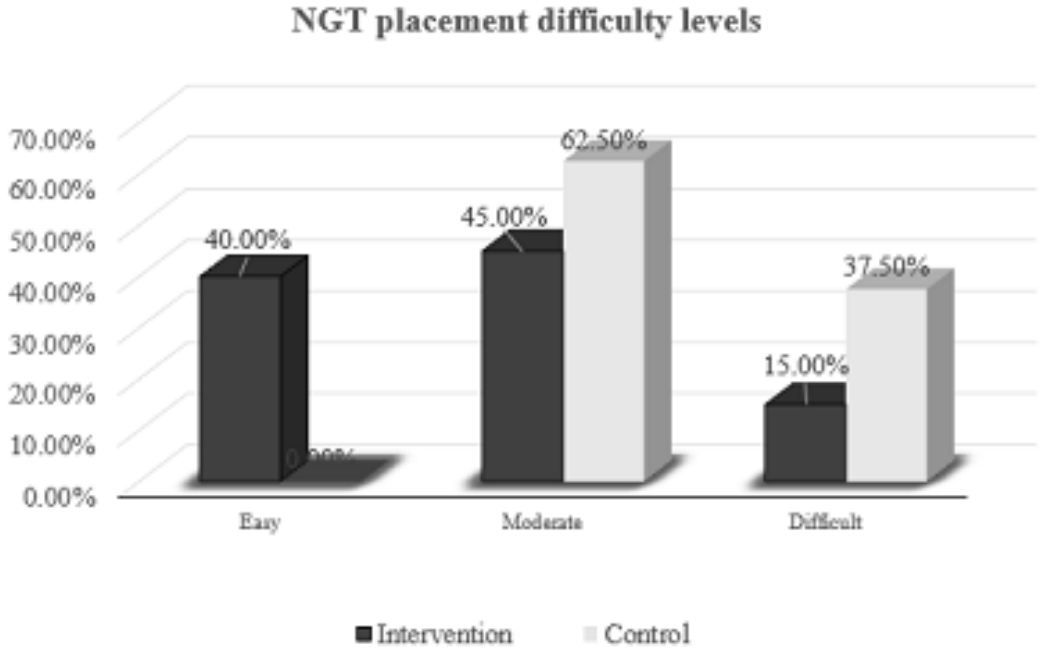

Figure 2: Difficulty levels of NGT placement.

similar to previous studies [13-15]. Based on the present study, the pain intensity could be reduced to $68 \%$ if an intravenous dose of metoclopramide had been administered $15 \mathrm{~min}$ before the NGT placement. This reduction was around $25-30 \%$ regarding experienced nausea and discomfort by patients. However, previous evidence showed that pain, nausea, and discomfort were reduced around $80-90 \%$, which is considerably higher than the findings of this study [14]. One possible explanation for this inconsistency may be the subjective measurement of study parameters and consequently a systematic overestimation of the parameters by patients in this study or underestimation by patients in the previous studies.

Findings the present study showed that pain in the intervention group in $T_{3}$ was around $54 \%$ of pain in $T_{1}$, while in the placebo group, it was around $60 \%$. Regarding nausea, this rate was around $50 \%$ in the placebo group and $27 \%$ in the intervention group, and in the case of discomfort around 66\% in the intervention group and $73 \%$ in the placebo group. The results of a study among critically ill patients revealed that using 20 mg of intravenous metoclopramide significantly improved the success rate of post-pyloric placement of spiral nasojejunal tubes. In this study, metoclopramide was administered 10 min before nasojejunal tube insertion [16]. However, another study does not confirm the efficacy of $10 \mathrm{mg}$ metoclopramide in improving post-pyloric placement of nasoenteral feeding tubes [17].

The emergency nurse who was responsible for the NGT placement reported that in the intervention group the insertion procedure was significantly easier compared 
with the placebo group. This finding could be considered as an intermediate reason for less pain, nausea, and discomfort in the intervention group. As a consequence of the more comfortable NGT placement, the probability of serious complications of the insertion is expected to be lower $[14,18,19]$. On the other hand, due to the crowding of the emergency departments [20], easier procedures may be highly valuable not only for patients but also for healthcare providers. Therefore, the use of intravenous metoclopramide before NGT insertion would lead to a higher quality of care in the emergency departments. Nevertheless, there are some limitations in the present study that need to be addressed. Although we tried to reduce the interpersonal variation during NGT insertion by using two experienced nurses, this method should be performed by different nurses on different patient populations to further generalize the results of this study.

\section{Conclusion}

In conclusion, according to the results of this study, it seems that using intravenous metoclopramide can significantly facilitate and improve the ease of NGT placement and reduce pain, nausea, and discomfort associated with NGT insertion in the emergency department.

\section{Acknowledgments}

The authors acknowledge the financial support of the deputy of research and technology of Mazandaran University of Medical Sciences.

\section{Ethical Considerations}

This study was approved by the institutional ethics committee of Mazandaran University of Medical Sciences (IR.MAZUMS.REC.94.1477). It was also registered under the Iranian Registry of Clinical Trials (IRCT2015083023696N2). The study objectives were explained to all participants. Informed consent was obtained from the participants and they also had the right to withdraw from the study at any time without penalty.

\section{Competing Interests}

None. 


\section{Funding}

This study has been financially supported by the Deputy of Research and Technology, Mazandaran University of Medical Sciences, Sari, Iran. Clinical trial registration number: IRCT2015083023696N2, https://www.irct.ir/.

\section{References}

[1] Metheny, N. A., Krieger, M. M., Healey, F., et al. (2019). A review of guidelines to distinguish between gastric and pulmonary placement of nasogastric tubes. Heart \& Lung, vol. 48, no. 3, pp. 226-235.

[2] Heidarzadi, E., Jalali, R., Hemmatpoor, B., et al. (2020). The comparison of capnography and epigastric auscultation to assess the accuracy of nasogastric tube placement in intensive care unit patients. BMC Gastroenterology, vol. 20, no. 1, pp. $1-6$.

[3] Yang, F. H., Lin, F. Y., and Hwu, Y. J. (2019). The feasibility study of a revised standard care procedure on the capacity of nasogastric tube placement verification among critical care nurses. Journal of Nursing Research, vol. 27, no. 4, p. e31.

[4] Walsh, S., Gallagher, A., Gallagher, R., et al. (2016). First do no harm: nasogastric tube placement and confirmation. Quarterly Journal of Medicine, vol. 109, no. 3, p. 219.

[5] Rouhi, A. J., Zeraatchi, A., Rahmani, F., et al. (2020) Effect of oral midazolam in pain relief of patients need nasogastric tube insertion: a clinical trial study. Journal of Research in Pharmacy Practice, vol. 9, no. 2, pp. 112-117.

[6] Liu, Y., Dong, X., Yang, S., et al. (2017). Metoclopramide for preventing nosocomial pneumonia in patients fed via nasogastric tubes: a systematic review and metaanalysis of randomized controlled trials. Asia Pacific Journal of Clinical Nutrition, vol. 26 , no. 5 , pp. $820-828$.

[7] Lubawski, J. and Saclarides, T. (2008). Postoperative ileus: strategies for reduction. Therapeutics and Clinical Risk Management, vol. 4, no. 5, p. 913-917.

[8] Kaneishi, K., Imai, K., Nishimura, K., et al. (2020). Olanzapine versus metoclopramide for treatment of nausea and vomiting in advanced cancer patients with incomplete malignant bowel obstruction. Journal of Palliative Medicine, vol. 23, no. 7, pp. 880881.

[9] Rumore, M. M., Lee, S. E., Wang, S., et al. (2011). Metoclopramide-induced cardiac arrest. Clinics and Practice, vol. 1, no. 4, pp. 174-178. 
[10] Ozucelik, D. N., Karaca, M. A., and Sivri, B. (2005). Effectiveness of pre-emptive metoclopramide infusion in alleviating pain, discomfort and nausea associated with nasogastric tube insertion: a randomised, double-blind, placebo-controlled trial. International Journal of Clinical Practice, vol. 59, no. 12, pp. 1422-1427.

[11] Bong, C. L., Macachor, J. D., and Hwang, N. C. (2004). Insertion of the nasogastric tube made easy. Anesthesiology, vol. 101, no. 1, p. 266.

[12] Durai, R., Venkatraman, R., and Ng, P. C. (2009). Nasogastric tube insertion technique and confirming position. Nursing Times, vol. 105, no. 16, pp. 12-13.

[13] Cullen, L., Taylor, D., Taylor, S., et al. (2004). Nebulized lidocaine decreases the discomfort of nasogastric tube insertion: a randomized, double-blind trial. Annals of Emergency Medicine, vol. 44, no. 2, pp. 131-137.

[14] Ozucelik, D., Karaca, M., and Sivri, B. (2005). Effectiveness of pre-emptive metoclopramide infusion in alleviating pain, discomfort and nausea associated with nasogastric tube insertion: a randomised, double-blind, placebo-controlled trial. International Journal of Clinical Practice, vol. 59, no. 12, pp. 1422-1427.

[15] Ducharme, J. and Matheson, K. (2003). What is the best topical anesthetic for nasogastric insertion? A comparison of lidocaine gel, lidocaine spray, and atomized cocaine. Journal of Emergency Nursing, vol. 29, no. 5, pp. 427-430.

[16] Hu, B., Ye, H., Sun, C., et al. (2015). Metoclopramide or domperidone improves postpyloric placement of spiral nasojejunal tubes in critically ill patients: a prospective, multicenter, open-label, randomized, controlled clinical trial. Critical Care, vol. 19, no. 1, p. 61.

[17] Heiselman, D. E., Hofer, T., and Vidovich, R. R. (1995). Enteral feeding tube placement success with intravenous metoclopramide administration in ICU patients. Chest, vol. 107, no. 6, pp. 1686-1688.

[18] Mandal, M. C., Dolai, S., Ghosh, S., et al. (2014). Comparison of four techniques of nasogastric tube insertion in anaesthetised, intubated patients: a randomized controlled trial. Indian Journal of Anaesthesia, vol. 58, no. 6, pp. 714-718.

[19] Pillai, J. B., Vegas, A., and Brister, S. (2005). Thoracic complications of nasogastric tube: review of safe practice. Interactive Cardiovascular and Thoracic Surgery, vol. 4, no. 5, pp. 429-433.

[20] Hosseininejad, M., Aminiahidashti, H., Pashaei, S. M., et al. (2017). Determinants of prolonged length of stay in the emergency department: a mixed method study from Iran. Emergency, vol. 5, no. 1, pp. 268-273. 